\title{
Influence of local heating on Marangoni flows and evaporation kinetics of pure water drops
}

Alexandros Askounis, ${ }^{*, 1,2}$ Yutaku Kita, ${ }^{1,2}$ Masamichi Kohno, ${ }^{1,2,3}$ Yasuyuki Takata, ${ }^{1,2,3}$ Vasileios Koutsos ${ }^{4}$ and Khellil Sefiane ${ }^{*, 4,5}$

${ }^{1}$ International Institute for Carbon-Neutral Energy Research (WPI-I'ICNER), Kyushu University, 744 Motooka, Nishiku, Fukuoka, 819-0395, Japan

2Department of Mechanical Engineering, Kyushu University, 744 Motooka, Nishi-ku, Fukuoka, 819-0395, Japan

${ }^{3}$ CREST, Japan Science and Technology Agency, 744 Motooka, Nishi-ku, Fukuoka 819-0395, Japan

${ }^{4}$ School of Engineering, The University of Edinburgh, King's Buildings, Mayfield Road, Edinburgh EH9 3JL, United Kingdom

${ }^{5}$ Tianjin Key Lab of Refrigeration Technology, Tianjin University of Commerce, Tianjin City, 300134, PR China

*To whom correspondence should be addressed: E-mail: a.askounis@i2cner.kyushu-u.ac.jp; Tel.: +81-

92-802-3905 Fax: +81-92-802-3905. E-mail: K.Sefiane@ed.ac.uk; Tel.: +44 (0)131 650 4873; Fax: +44

(0)1316506551

\begin{abstract}
The effect of localized heating on the evaporation of pure sessile water drops was probed experimentally by a combination of infrared thermography and optical imaging. In particular, we studied the effect of three different heating powers and two different locations, directly below the center and edge of the drop. In all cases, four distinct stages were identified according to the emerging thermal patterns. In particular, depending on heating location, recirculating vortices emerge which either remain pinned or move azimuthally within the drop. Eventually, these vortices oscillate in different modes depending on heating location. Infrared data allowed extraction of temperature distribution on each drop surface. In turn, the flow velocity in each case was calculated and was found to be higher for edge heating, due to the one-directional nature of the heating. Additionally, calculation of the dimensionless Marangoni and Rayleigh numbers yielded the prevalence of Marangoni convection. Heating the water drops also affected the evaporation kinetics by promoting the "stick-slip" regime. Moreover, both the total number of depinning events and the pinning strength was found to be highly dependent on heating location. Lastly, we report a higher than predicted relationship between evaporation rate and heating temperature, due to the added influence of the recirculating flows on temperature distribution and hence evaporation flux.
\end{abstract}

Keywords: drop evaporation, laser heating, Marangoni flows, evaporation kinetics, infrared thermography 


\section{Introduction}

The evaporation of sessile, liquid drops has attracted considerable scientific attention due to its importance in a wide range of biological and technological applications. Inkjet printing, ${ }^{1,2}$ DNA mapping, ${ }^{3,}$ ${ }^{4,5}$ biomedical diagnosis, ${ }^{6,7,8}$ and surface patterning ${ }^{9,10,11}$ are but a few of the areas which may benefit from advancing the understanding of the process. However, the interplay among fluid dynamics, heat and mass transfer and liquid-surface interactions complicates the complete comprehension of drop drying.

A sessile drop evaporating freely on a hydrophilic surface (water contact angle $<90^{\circ}$ ) will exhibit a higher evaporation rate at the three-phase contact line $(T L),{ }^{12}, 13,14$ which induces an outward fluid flow to replenish the evaporated fluid. In the presence of particles, this outward flow carries particles from the bulk drop to the TL where they deposit and form a "coffee-stain" deposit. ${ }^{15,}{ }^{16}$ At the same time, a temperature gradient is formed on the surface of the drop due to either evaporative cooling ${ }^{17,18}$ or heat conduction via the substrate ${ }^{13,19}$ This temperature gradient, in turn, may induce temperature dependent recirculating flows such as buoyancy driven Rayleigh ${ }^{20,21,22}$ or surface tension driven Marangoni. ${ }^{14,23,24}$

Visualization of these convective flows has been reported mainly for volatile liquids such as alcohols and refrigerants induced by high temperature gradients and possibly due to the fact that they tend to attract small amount of contaminants in comparison with water. For example, recirculating vortices in hanging methanol drops were attributed to Rayleigh convection. ${ }^{25}$ Elsewhere, alcohol and refrigerant drops were shown to exhibit a number of thermal/liquid waves moving in the azimuthal direction, which were coined hydrothermal waves (HTWs). ${ }^{26,27,28} \mathrm{HTW}$ s were attributed to Marangoni stresses. Numerous theoretical works were conducted in order to shed light on the underpinning physics of the internal flows in volatile drops and are now considered well understood. ${ }^{29,} 30,31,32$ 
On the contrary, the internal flows emerging in a drying water drop have received rather limited attention. Marangoni flows, in particular, remain a controversial issue. In fact, theoretical approximations predict strong Marangoni flows in water drops, ${ }^{13,32,33}$ which have not been corroborated experimentally due to water being prone to contaminants. ${ }^{15,}{ }^{34}$ The contaminants should interfere with the surface tension gradients, responsible for Marangoni recirculation, and lead to a uniform thermal distribution on water drop surface, as reported with infrared thermography measurements. ${ }^{26,27}$ In a previous work, we reported the first experimental induction and visualization of Marangoni flows in pure water drops with localized heating directly below the center. ${ }^{35}$ The contact angle of the drops was $c a .104^{\circ}$ which is predicted to lead to a homogenous evaporative flux and hence a uniform temperature distribution across the drop surface ${ }^{36}$ Therefore, localized heating was capable of generating sufficient thermal gradient and in turn surface tension gradient to overcome the negating effect of the contaminants. At the same time, the temperature gradient interferes with the evaporative cooling of the drop, the main mode of heat transfer in drying drops, ${ }^{18,37}$ resulting in the induction of Marangoni flows in an attempt to homogenize the temperature distribution in the drop. However, this contribution was limited in demonstrating the existence of Marangoni flows in pure water drops.

In the present work, we attempt a systematic analysis of the effect of local heating on a drying drop of pure water. In particular, we investigate how heating the edge and the center of the drop at three different heating powers interferes with the predicted homogenous temperature distribution across the surface $^{36}$ and in turn influences the emerging Marangoni flows. The flow pattern evolution and Marangoni flow velocity was found to be dependent mainly on heating location and to a lesser extent on heating power. Furthermore, we provide a comprehensive comparison of the evaporation and TL kinetics where local heating leads to "stick-slip" evaporation. Unexpectedly, heating location was found to determine the 
number of TL depinning events with more events occurring as the edge heating power increased and fewer events as the center heating power increased. Quantification of the pinning barrier allowed us to deduce that edge heating imposed a higher temperature which lowered the surface tension and hence led to lower pinning barrier. The evaporation kinetics was also found to be highly affected by localized heating, with evaporation rate exhibiting a dependence on temperature in the form of a power cube relation, compared to the $3 / 2$ exponent of the diffusion model.

\section{Experimental Section}

$10 \mu \mathrm{L}$ drops of deionized water were gently deposited on $50 \mu \mathrm{m}$ thick copper substrates covered with a thin, $20 \mathrm{~nm}$, Cytop (C) layer in order to control drop shape. Initial contact angle and radius of the drops were $c a .104^{\circ}$ and $1.4 \mathrm{~mm}$, respectively. The contact angle hysteresis was measured to be $19^{\circ}$. Local heating of the substrate directly below the center and the edge of the drop was realized using an IntegraMP-30WW diode laser (808 nm wavelength, Spectra-Physics) operating in continuous wave mode. Laser power was kept constant at $0.3 \mathrm{~W}, 1.9 \mathrm{~W}$ and $3.4 \mathrm{~W}$, as measured with a laser power meter (Vega, Ophir Optronics Solutions Ltd.). A combination of an infrared (top view, temperature resolution of $18 \mathrm{mK}$, SC4000, FLIR) and a CCD (side view, resolution of $10 \mu \mathrm{m} / \mathrm{px}$, STC-MC152USB, Sentech) camera was employed for the simultaneous acquisition of the temporal evolution of the thermal patterns and the profile of the drops. Using custom built Matlab code, we extracted the drop profiles (contact angle, radius and volume) from the optical videos (framerates of $4.75 \mathrm{fps}$ ). Additionally, the minimum and maximum temperature, $T_{\min }$ and $T_{\max }$, respectively, were extracted for each drop from the IR videos (framerates of 5, 30 and $60 \mathrm{fps}$ for each laser power), using a second custom built Matlab code. Maximum drop temperature was found to range $30^{\circ}<T_{\max }<80^{\circ} \mathrm{C}$ from lowest to highest heating. Figure 1 depicts the effect of laser heating the substrate. Dashed lines in the bottom row indicate the position of the drop circumference in relation to the imposed hot-spot. All the experiments were carried out in an 
environmental chamber (PR-3KT, ESPEC Corp.) to keep temperature and relative humidity at $18.0 \pm 0.5^{\circ} \mathrm{C}$ and $40 \pm 10 \%$, respectively. Experiments were repeated at least 10 times and showed good reproducibility.

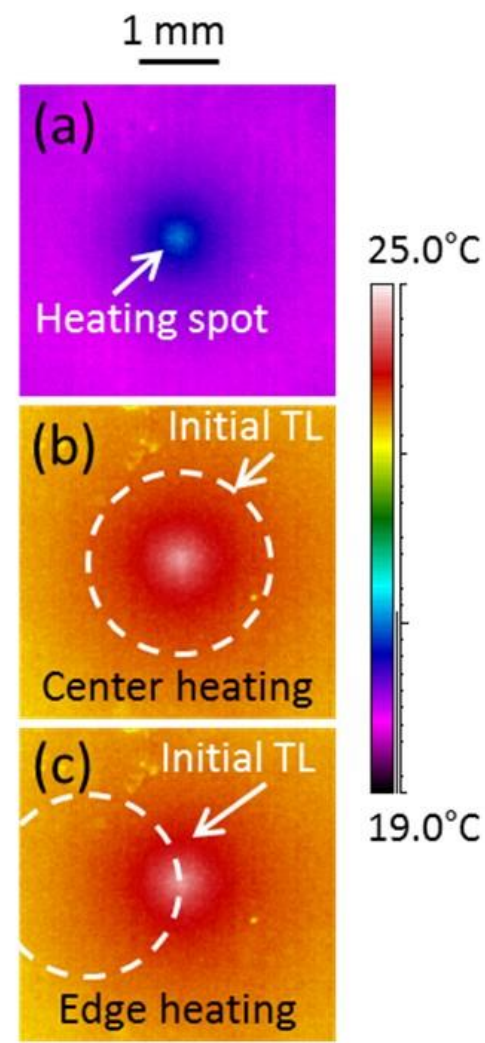

Figure 1: Sample IR images showing the imposed temperature gradient on a bare substrate at $0.10 \mathrm{sec}$ after heating was initiated. (b) and (c) were taken at 1.80 seconds and dashed lines show the circumference of a $10 \mu \mathrm{L}$ drop for center and edge heating, respectively.

\section{Results and discussion}

First, we focus our attention on how local heating of the substrate directly below the center and at the edge of a pure water drop using three different levels of laser power affected the internal flows. Water is opaque in the spectral range of our camera, hence the IR camera is capable of capturing the spatiotemporal evolution of the thermal distribution on the drop surface. We present and discuss only 
the first moments of the evaporation, when the droplet is pinned. All thermal patterns exhibit the same behavior throughout the rest of the process.

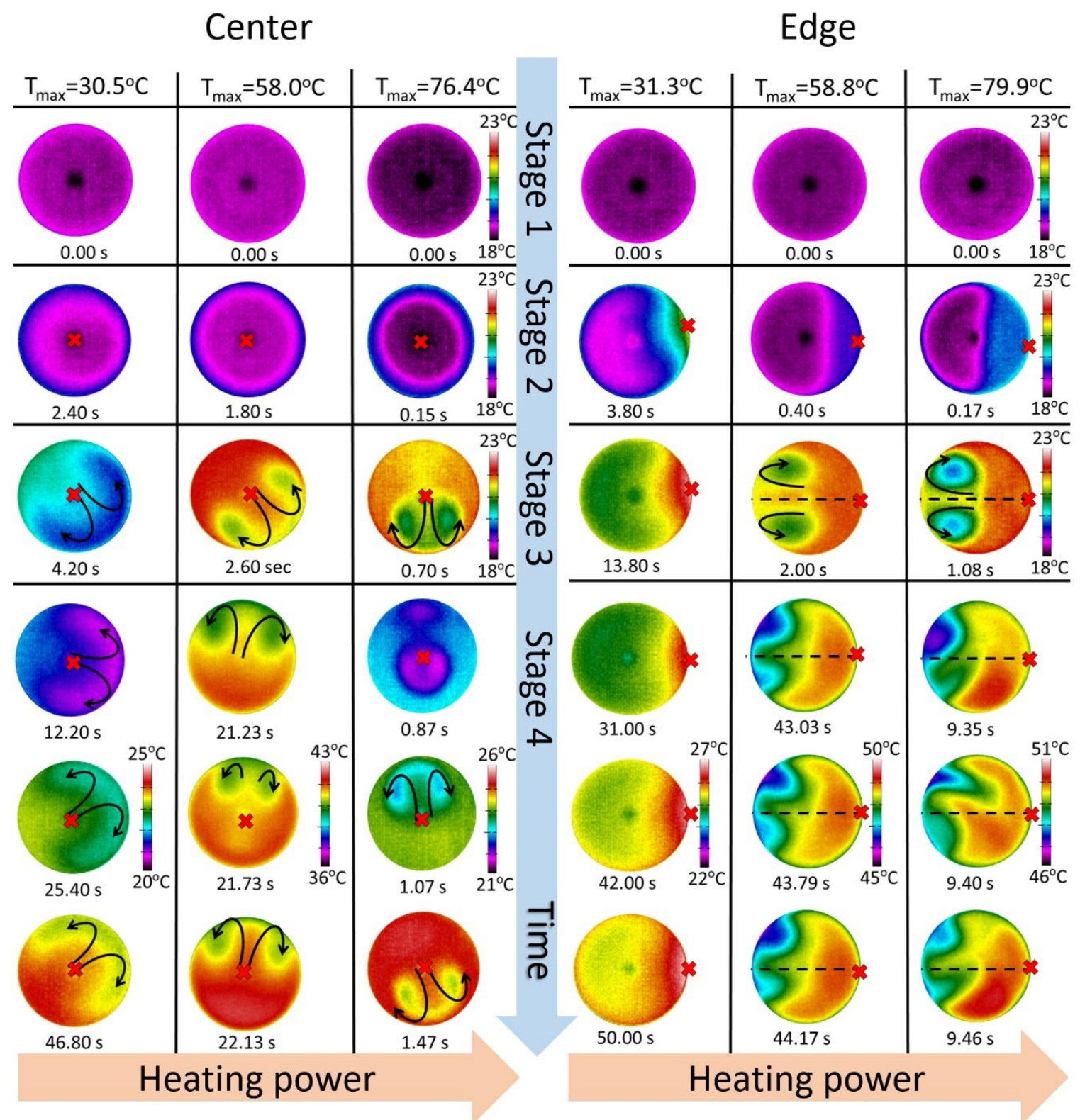

Figure 2: Comparison of heating location and power effect on the emergence and first moments of evolution of the thermal patterns in pinned water drops. 
Center heating. The IR images in the left side of Figure 2 depict the evolution over time of the thermal patterns arising within the water drops when heating them directly below the center at different powers from left to right. The images are divided in four distinct stages for better understanding. During Stage 1, every drop is evaporating without heating and the IR images of all cases depict a uniform thermal distribution, in agreement with previous reports. ${ }^{26,29}$ The darker dot at the center of each drop is the reflection of the camera and hence is neglected. Heating is initiated in Stage 2 which induces a ring-like heating pattern at the periphery of the drop, due to heat transfer from the substrate to the liquid-air interface of the drop, in agreement with the literature..$^{29,32,35}$ As the convective flows set in, recirculating flows emerge manifesting as twin vortices (Stage 3). Essentially, the vortices are formed when heat from the heating spot travels within the drop until arriving at the liquid-air interface, at which point the flow recirculates to the center in an attempt for the system to attain thermal equilibrium. Eventually, each drop evaporation enters Stage 4, at which point the thermal gradients become highly irregular leading to different thermal patterns. In the low power case $\left(T_{\max }=30.5^{\circ} \mathrm{C}\right)$, the recirculating front moves azimuthally. The recirculating front in the medium case $\left(T_{\max }=50.6^{\circ} \mathrm{C}\right)$ exhibits a similar azimuthal motion until the minimum drop temperature, $T_{\min }$, reaches $c a .40^{\circ} \mathrm{C}$, at which point the pair of the twin vortices begin an oscillating merging and splitting, in agreement with previous report. ${ }^{35}$ On the other hand, the high heating case $\left(T_{\max }=76.4^{\circ} \mathrm{C}\right)$ exhibits a periodic shift of the recirculation front from one side of the drop to the other. As with the emergence of the flows, this periodic shift of the recirculating front could be attributed to temperature difference this time between the two hemispheres of the drop. As observed, in the IR images, the temperature difference between the two hemispheres increases during heating and leads to a large surface tension gradient capable of shifting the front. This fact is further supported by our calculation of the timescale of the phenomenon in the following section about the temperature gradient oscillations. 
Edge heating. In addition to heating power, we also investigated the effect of the location of the heating spot on the emerging thermal patterns. To this end, the edge of each drop was heated locally, as depicted in the right side of Figure 2, with increasing power from left to right. In this case, Stage 1 corresponds to no heating, which is readily apparent given the uniform thermal distribution in all cases. Stage 2 corresponds to the onset of heating, which can be identified as the hotter region to the right of each image. As thermal gradients increase, convective flows emerge and manifest in vortices (Stage 3) with their strength related to heating power. Notably, the heating in the first column is insufficient to induce vortices and the thermal motion in this case progresses in the same manner. The other two cases, however, follow a similar behavior when entering Stage 4. In this Stage, the vortices remain at the same location without the azimuthal motion identified for center heating in Figure 2. Furthermore, in both cases, when $T_{\min }$ reaches $c a .45^{\circ} \mathrm{C}$, the vortices become unstable and their strength begins to fluctuate. In particular, the strength of one vortex increases while simultaneously the other one decreases in a sequential manner. This fluctuation leads to an oscillation of the flow around the main heat conduction path, dashed lines in Figure 2. This oscillation could be ascribed to the difference in the thermal distribution between the two hemispheres defined by the dashed lines, which result in a surface tension difference and the observed flows. The difference in temperature between the two hemispheres could perhaps be attributed to a surface defect interfering with heat conduction or a small, non-detectable asymmetry in drop shape.

Temperature gradient $(\Delta \mathbf{T})$ variations within the drops. Further analysis of the thermographic images in Figure 2 allows us to plot the temporal variations of $\Delta T$ for 60 seconds of each drop. Results are plotted in the left column of Figure 3, categorized with increasing heating power from top to bottom panel. At the onset of heating in Figure $3(\mathrm{a})$, a $\Delta T \sim 1.5^{\circ} \mathrm{C}$ is required to induce vortices for center heating (black line), whereas a higher $\Delta T \sim 2.5^{\circ} \mathrm{C}$ appears to be insufficient for edge heating (red) case. Eventually both cases 
exhibit a similar behavior where $\Delta T$ decreases and remains virtually constant. On the other hand, in Figure 3 (c), an initial increase $\Delta T \sim 2.0^{\circ} \mathrm{C}$ results in the emergence of the vortices for both center (black) and edge (red) heating. Eventually, the center heated case (black line) exhibited an oscillating behavior which corresponds to the observed merging and splitting of the vortices (Figure 2), as discussed previously. ${ }^{35}$ On the contrary, during edge heating, $\Delta T$ appears to increase until reaching a plateau at $\Delta T \sim 2.5^{\circ} C$ and at ca. $40 \mathrm{~s}$ an oscillation emerges, corresponding to the periodic increase in the strength of one vortex with a decrease in the other one and oscillation of the recirculating front around the main heat path shown with dashed line in Figure 2. Lastly, in Figure 3(e), both cases exhibit a slightly higher initial requirement, $\Delta T \sim 2.5^{\circ} C$, for the onset of recirculation. Eventually, $\Delta T$ begins to oscillate around a constant value of 1.9 and $2.5^{\circ} \mathrm{C}$ for center and edge heating respectively. The observed oscillation corresponds to the sequential shift of the recirculating front from one side of the drop to the other for center heating (black line) and around the main heat flow path for edge (red line) heating. The corresponding fast Fourier transformation (FFT) of the $\Delta T$ temporal evolutions during Stage 4 is presented in the right column of Figure 3. No distinguishable peak either for center or edge heating can be identified in Figure 3(b). On the other hand, increasing the heating power results in the emergence of a major peak at approx. 1.23 and a secondary one at $2.51 \mathrm{~Hz}$ for center heating (black line) whereas edge heating (red line) exhibits a small peak at $7 \mathrm{~Hz}$, as shown in Figure 3 (d). Further increase in heating power (Figure 3 (f)), leads to a dominant frequency at $2.46 \mathrm{~Hz}$ for center heating (black line) and to two similar in amplitude/power frequencies at 2.77 and $11.72 \mathrm{~Hz}$ for edge heating (red line). These frequencies should be related to the characteristic timescale of the Marangoni flows. To verify this, we initially calculate the Marangoni time $\tau_{M a}=$ $\left[\rho R H^{2} /(\beta \Delta \mathrm{T})\right]^{1 / 2}$ and then estimate the characteristic Marangoni frequency $f_{M a}=1 / \tau_{M a} \sim 9 H z,{ }^{40}$ which is in the same order of magnitude as the values reported in Figure 3. 

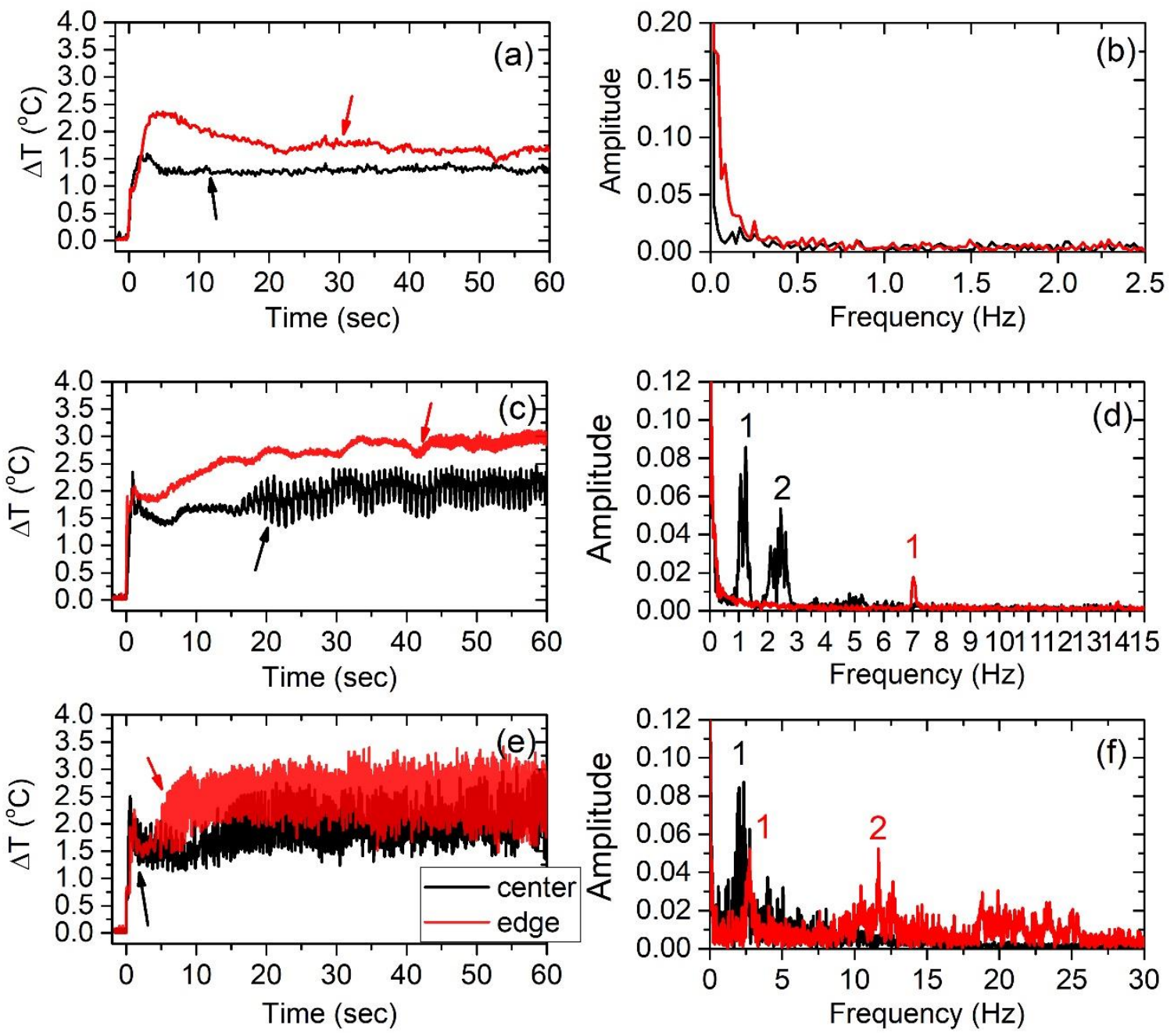

Figure 3: (left) Variation of interfacial temperature difference, $\Delta T$, as a function of time for heating at the edge (red) and center (black). Arrows indicate the beginning of Stage 4. (right) corresponding FFT analysis of $\Delta T$ oscillation during Stage 4 for each case. Heating power increases from top to bottom row. In all cases, laser irradiation is initiated at $0.0 \mathrm{~s}$ and heating power increases from top to bottom rows.

Using this information, we attempt, here, to determine the origin of the above flows. Garnier et al., has established the criterion of the ratio of the dimensionless Marangoni (thermocapillary-driven) over Rayleigh (buoyancy-driven) numbers $\chi=R a / M a$ for a liquid disk, ${ }^{41}$ which was later adopted for drops by Sefiane et al. ${ }^{26}$ Rayleigh number is given as $R a=\beta g H^{4} \Delta T / v \alpha R$ and Marangoni as $M a=\gamma H^{2} \Delta T / \rho v \alpha R$, where $R$ and $H$ are characteristic radial and vertical length, respectively, $\beta$ is the thermal expansion 
coefficient, $\gamma$ is the surface tension, $\rho$ is the density, $v$ the kinematic viscosity and $\alpha$ the thermal diffusivity. The average values of $R a, M a$ and $\chi$ are presented in Table 1 along with the Bond (Bo) number for comparison. In all cases, $R a / M a \ll 1$ which combined with the fact that the $R a$ in every case remains below the critical value of $1000,42,43$ allows us to safely conclude that the observed flows are Marangoni in origin. Furthermore, the emergence of these Marangoni flows should be an attempt of the system to attain thermal equilibrium by homogenizing the heat and/or temperature within the drop.

\begin{tabular}{|c|c|c|c|c|c|}
\hline $\begin{array}{l}\text { Heating } \\
\text { location and } \\
T_{\max }(\mathrm{K})\end{array}$ & $\Delta T$ & $\mathrm{Ra}$ & $\mathrm{Ma}$ & $\chi$ & Bo \\
\hline Center - 30.5 & 1.38 & 190 & 3700 & 0.046 & 0.44 \\
\hline Center - 58.0 & 1.63 & 600 & 7800 & 0.076 & 0.42 \\
\hline Center - 76.4 & 1.97 & 700 & 8800 & 0.082 & 0.38 \\
\hline Edge -31.3 & 1.88 & 180 & 4600 & 0.046 & 0.4 \\
\hline Edge - 58.8 & 1.94 & 680 & 10100 & 0.072 & 0.39 \\
\hline Edge -79.9 & 2.22 & 950 & 11000 & 0.081 & 0.38 \\
\hline
\end{tabular}

Table 1: Effect of heating location and power on $\Delta T, R a, M a, \chi$ and $B o$.

As the Marangoni flows are an attempt to homogenize the heat and/or temperature within the drop, then the strength of the vortices should be a function of the heating power. Focusing our attention on Stage 3 in Figure 2, we may stipulate that the recirculating flow velocity is dependent on the imposed heating. To quantify this relationship we calculated the recirculating flow velocity, $V_{M a}$, which scales with the temperature gradient $\Delta T=T_{\max }-T_{\min }$, as $V_{M a}=(1 / 32)\left(\beta \theta^{2} \Delta T / \mu\right){ }^{13,}{ }^{38}$ where the surface tension 
gradient with respect to temperature is given by $\beta=d \gamma / d T$ (in the present case $\beta=-1.68 \times$ $\left.10^{-4} \mathrm{~N} / \mathrm{m} \cdot \mathrm{K}\right){ }^{39} \theta$ denotes the contact angle and $\mu$ the dynamic viscosity (in the present case $\mu=1.0 \times$ $\left.10^{-3}(N \cdot s) / m^{2}\right) \cdot{ }^{39}$ The $\Delta T$ values used are given in Table 1 and were extracted from IR data and $\theta$ values from the CCD data. Results are plotted in Figure 3 (a) as a function of $T_{\max }$ and were found to be in line with the literature. ${ }^{39}$ The effect of heating location on $V_{M a}$ is readily here and found to be stronger for edge heating, where it induces stronger temperature gradient, $\Delta T$, across the drop surface. To further highlight this effect we plotted $V_{M a}$ as a function of $\Delta T$ in Figure 3 (b). $\Delta T$ and its effect on the thermal patterns is discussed in depth in the following section.

(a)

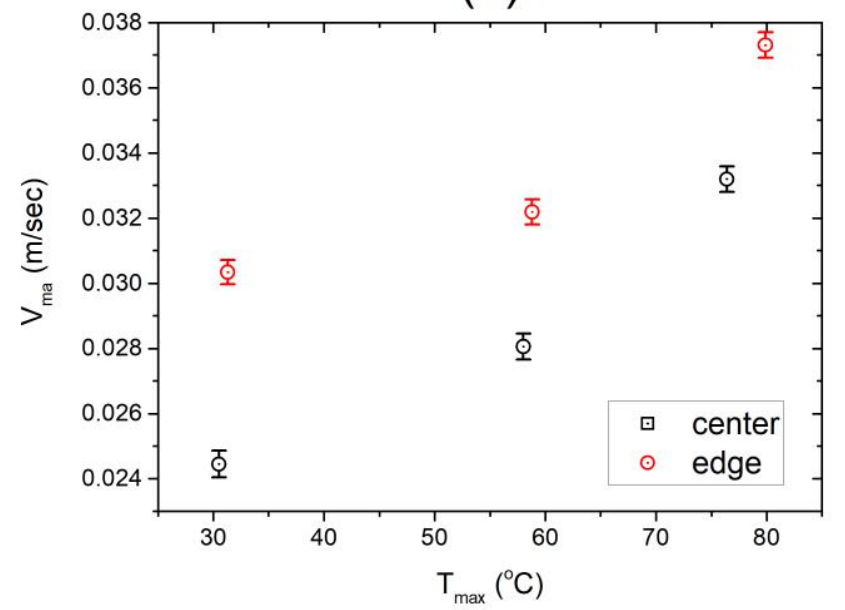

(b)

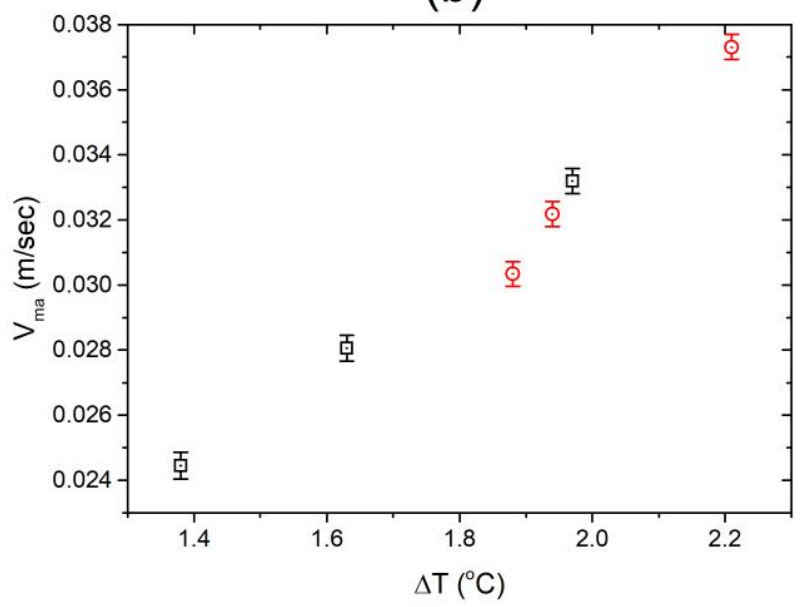

Figure 4: Calculated flow velocity, $V_{M a}$, as a function of (a) $T_{\max }$ and (b) $\Delta T$.

Triple line motion kinetics. The drops in all the cases presented herein exhibited "stick-slip" evaporation, as presented in the plots of contact radius, $R$, in Figure $5(\mathrm{a}, \mathrm{b}, \mathrm{c})$ and contact angle, $\theta$, Figure $5(\mathrm{~d}, \mathrm{e}, \mathrm{f})$ as a function of time and with increasing power from left to right. Additionally, the effect of heating at the edge and center are plotted in each panel with red and black lines for comparison. "Stick-slip" evaporation consists of TL pinning, during which $R$ remains virtually constant with decreasing $\theta$, interrupted by 
instantaneous depinning events which may be identified by a rapid drop in $R$ and an increase in $\theta$ (for details in "stick-slip" mechanism see Ref. ${ }^{44}$ ).
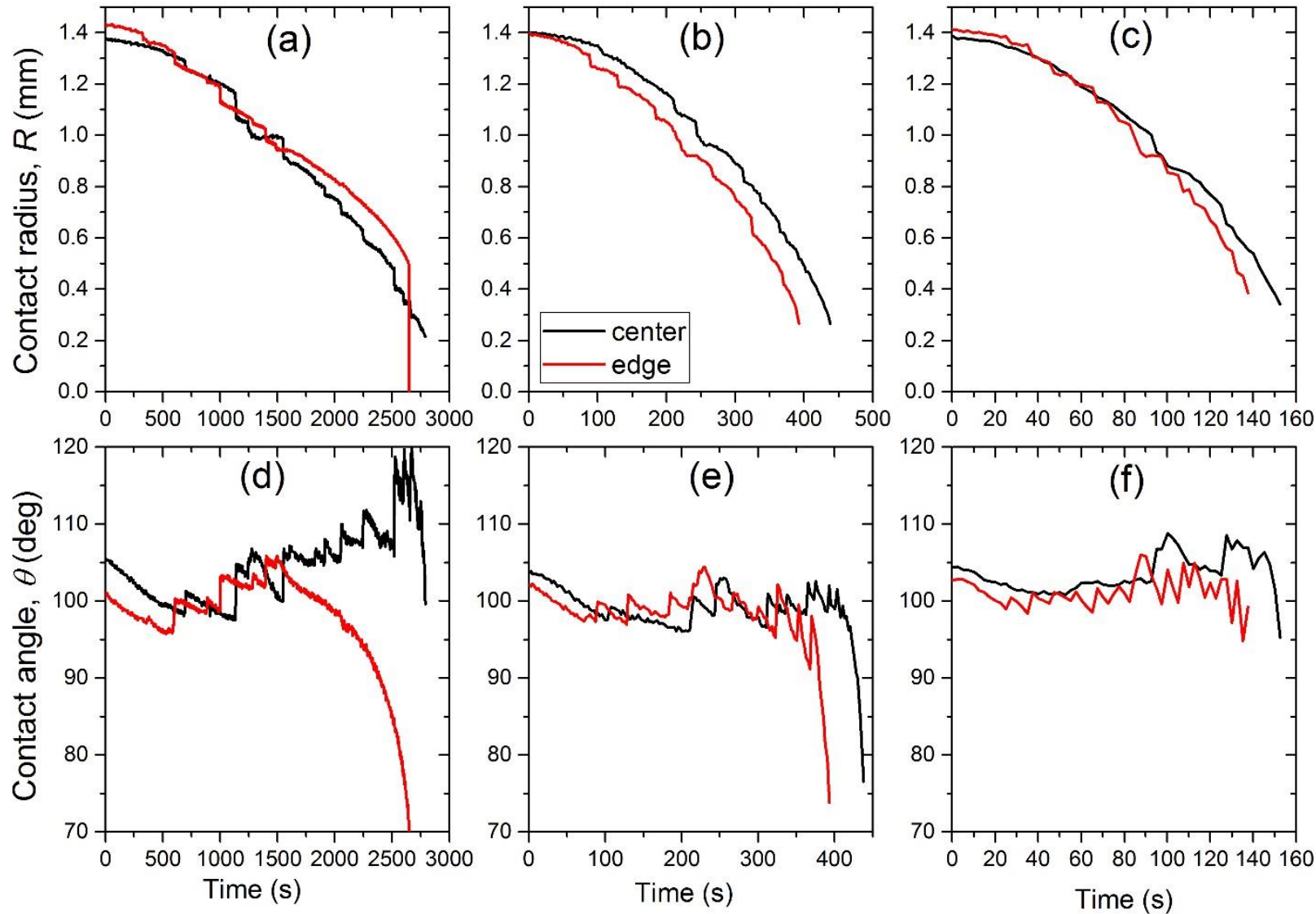

Figure 5: Evolution over time of (top) contact radius, $R$, and (bottom) contact angle, $\theta$, for increasing heating power from left to right.

The results shown in the top row of Figure 5 provide only a side-view of the TL motion events. To alleviate this deficiency, we provide in Figure 6 a schematic illustration of the TL location during each pinning event as acquired from the top view of the IR camera. From this simple illustration we may extract further information about the effect of, mainly, heating location on the TL motion kinetics of each drop. In more detail, at low heating power both center and edge heating exhibit a negligible effect on TL motion. 
Increasing the power results in a noticeable difference. In particular, the TL at the edge heating case exhibits a preferential depinning and moves away from the heating source. In addition, the highest power case in center heating exhibits a similar behavior. This could be attributed to the preferential depinning during stick-slip evaporation, ${ }^{45}$ which brings the edge of the droplet near or on top of the hot spot leading to edge heating. This preferential depinning could be surface tension driven. As explained above, a surface tension gradient is generated on the drop surface due to localized heating. In a similar manner, edge heating should result in a, local, lower interfacial tension there compared to the cold side. In turn, this surface tension gradient should allow the TL near the hot source to depin and move away from the hot source. A similar thermocapillary driven motion was reported to occur when a water drop was placed on the hot side of an oil-impregnated surface (contact angle hysteresis, $\left.\mathrm{CAH} \sim 1^{\circ}\right) .{ }^{46} \mathrm{~A}$ second potential and complementary depinning mechanism could be the continuous, one-directional Marangoni flow, carrying liquid from the hotter to the colder side. In turn, the amount of liquid on the hot side should deplete faster and lead to more depinning events occurring at that side. 


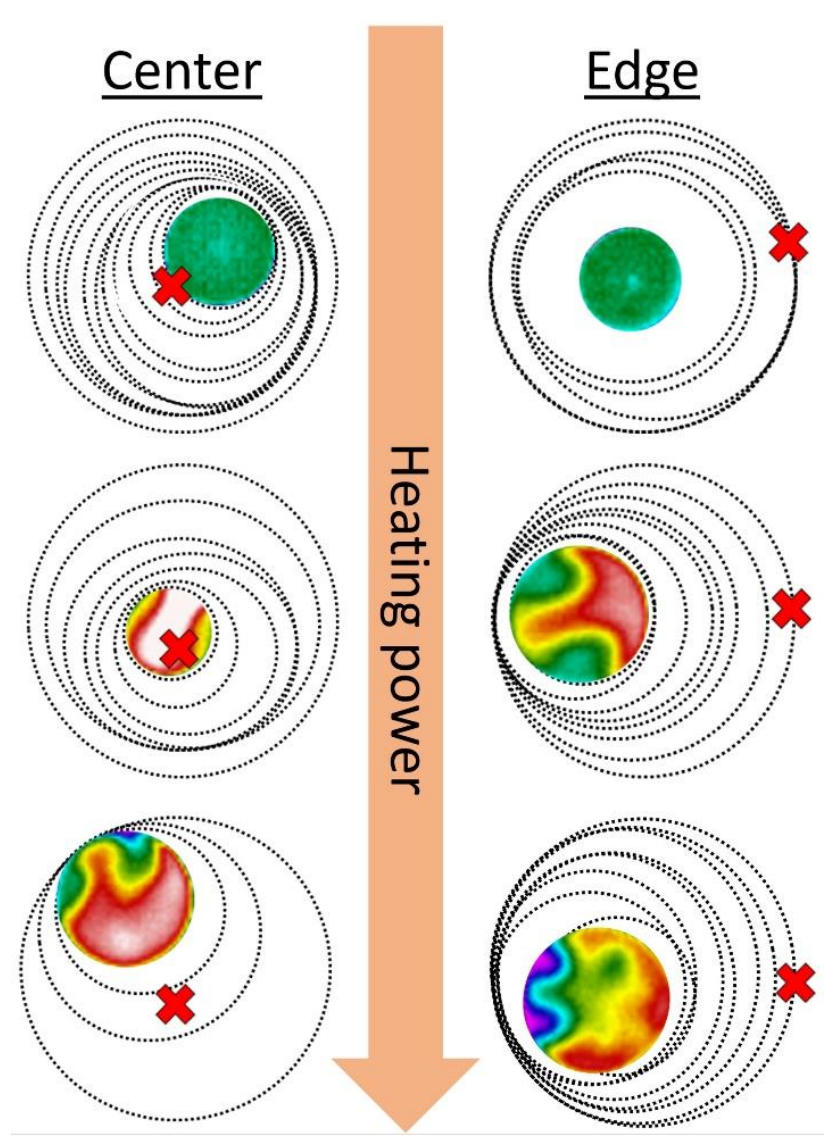

Figure 6: Schematic illustration of TL pinning locations with dashed lines, as extracted from the experimental IR data for all drops presented above. Initial drop diameter in every case is approx. $3 \mathrm{~mm}$. Colored circles are temperature maps of the final pinning event and crosses demonstrate the heating location.

Figure $7(a)$ depicts the number of depinning events as a function of $T_{\max }$, as extracted from the top view of IR images. In the center heating case (black line) increasing the heating power reduces the number of depinning events. At first, low heating, could provide the system enough energy to overcome the pinning barrier in a similar manner as lowering the ambient pressure. ${ }^{45}$ However, stronger and oscillating Marangoni flows emerge at higher temperatures. Hence, these depinning events could perhaps be attributed to the emerging flow patterns replenishing faster the liquid across the TL and thus retarding depinning, to an extent. On the other hand, in edge heating (red line) increasing heating power results in a higher number of depinning events. This behavior could be attributed to the one-directional (from the 
heating spot to the right to the cold spot to the left) nature of the emerging Marangoni flows which only oscillate around the central, hotter heating path, shown with the dashed lines in Stage 4 of Figure 2. Essentially, this heating pattern induces a continuous flow from the hotter side of the drop to the colder. Hence, the amount of liquid on the hot side should be depleting constantly and eventually result in more depinning events occurring at that side for continuity. Furthermore, edge heating should provide the drop with more energy to depin, leading to more rapid "stick-slip" cycles and lower hysteretic energy barrier pinning each drop.

Let us at this point quantify the effect of localized heating on the excess free energy of the drop, due to the drop being out of equilibrium. Essentially, the shape of a sessile drop at equilibrium should correspond to a minimum of all the forces acting on the TL and may be represented by Young's angle, $\theta_{0}$. Should the drop shape deviate from, $\theta_{0}$, by an infinitesimally small value, $\delta \theta$, then the free energy of the drop should increase above its minimum value at equilibrium. Once this increase exceeds the wetting barrier (unique to each three-phase, $T L$, combination), the TL depins. Here, we quantify the excess free energy of the drop as a function of contact angle change, $\delta \theta=\theta_{0}-\theta$. The excess free energy normalized per TL unit length, $\delta \bar{G}$, may be expressed as: $:^{44,45,47}$

$\delta \bar{G}=\frac{\gamma R(\delta \theta)^{2}}{2\left(2+\cos \theta_{0}\right)}$

where, $\gamma$ is the liquid surface tension measured using the pendant drop technique and found to be $c a$. $0.073 \mathrm{~N} / \mathrm{m} . R$ and $\theta$ are the values of contact radius and angle, respectively, extracted from Figure 5 . The energy requirement for the first depinning event in every drop was calculated using Eq. 1 and results are plotted in Figure 7 (b). The $\delta \bar{G}$ values vary widely, from ca. $14 \times 10^{-7}$ to $3 \times 10^{-7} \mathrm{~N}$ for center and edge heating, respectively. It is readily apparent that edge heating results in smaller pinning barriers, 
which could be attributed to the fact that the surface tension of water is inversely proportional to temperature. Hence, smaller $\delta \bar{G}$ should be required for drops to depin at higher temperatures. In addition, $\delta \bar{G}$ at edge heating is $\sim 50 \%$ smaller than center heating, perhaps due to the smaller amount of hot area covered by the drop. In the schematic representation in Figure 1 (c), the drop covers all of the hot area and most of the heat generated locally should be conducted by the drop. On the other hand, only a small portion of the hot area is covered from the drop in the edge heating case, as represented in Figure 1 (d). Therefore, the temperature should be higher locally in the edge case, leading to a lower surface tension and, in turn, to lower $\delta \bar{G}$.

(a)

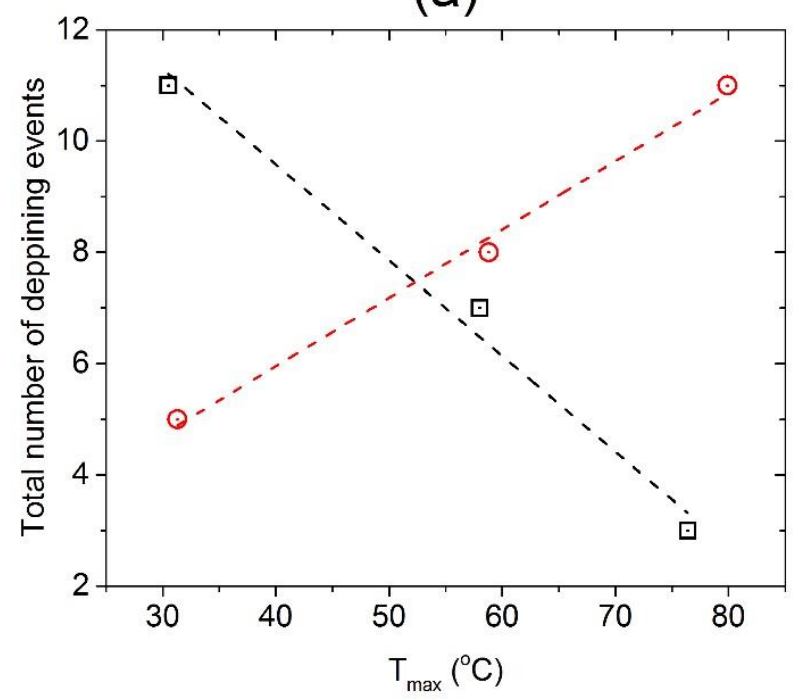

(b)

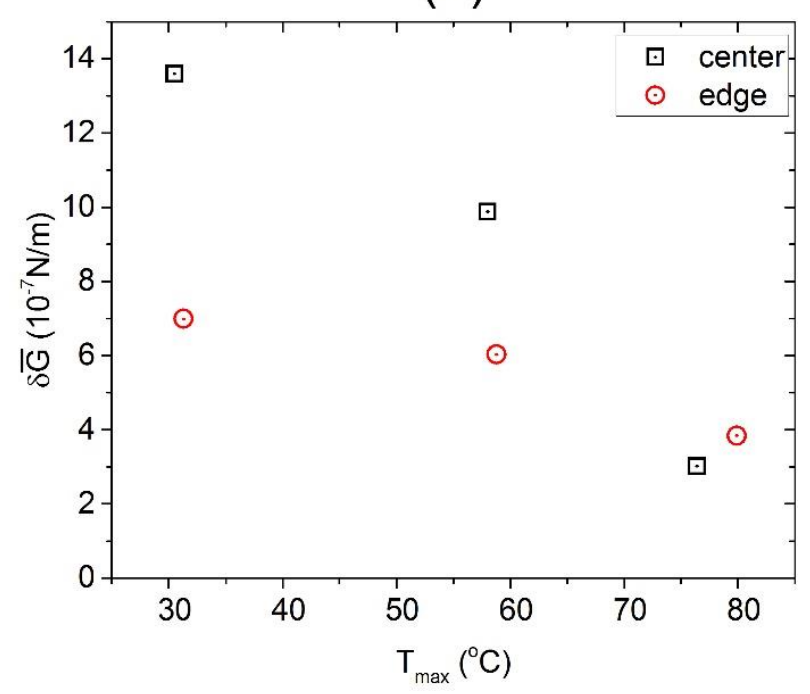

Figure 7: (a) Total number of depinning events as a function of heating power for center (circles) and edge (squares) heating. (b) Energy requirement, $\delta \bar{G}$, for first depinning to occur. Dashed lines are guide to the eye.

Evaporation kinetics. Figure 8 (a) depicts the depletion of normalized volume as a function of normalized time for all drops in logarithmic scale. A crude comparison of these curves indirectly yields a nonlinear relationship between the average evaporation rate and the heating power. However, the heating location 
effect is not evident here. From the data in Figure 8 (a), we determined the average evaporation rate, $-d V / d t$. Results are plotted in Figure 8 (b) with squares for center and circles for edge heating and two distinct trends emerge revealing the significance of heating location. The relationship of $-d V / d t$ and temperature may be described as $-d V / d t=a T_{\max }^{b}$, with a fitting parameter $b=16.73 \pm 1.69$ and $17.93 \pm 1.06$ for edge and center heating, respectively. Generally, the average evaporation rate, $-d V / d t$ is given by: ${ }^{33,48}$

$-\frac{d V}{d t}=\frac{\pi R D c_{s a t}(1-R H)}{\rho} f(\theta)$

where $R$ is the contact radius, $\rho$ is the density of water, $R H$ is relative humidity and $f(\theta)$ a complex function of contact angle given for the range of initial contact angles $10^{\circ}<\theta<180^{\circ}$ and given in detail in Ref. ${ }^{49}$. The diffusion coefficient, $D$, and the saturated vapor concentration near the drop, $c_{s a t}$, are both temperature dependent on the nature, pressure or temperature of the gas. According to Poling et al., $D$ for a binary system can be expressed as: ${ }^{50}$

$D=\frac{0.00266 T^{3 / 2}}{P M_{A B}^{1 / 2} \sigma_{A B}^{2} \Omega_{D}}$

where $\sigma_{A B}$ is the characteristic Lennard-Jones length, $\Omega_{D}$ is the diffusion collision integral, $M_{A B}$ is the molecular weight of gas $\mathrm{A}$ and $\mathrm{B}$ related as $M_{A B}=2\left[\left(1 / M_{A}+1 / M_{B}\right)\right]^{-1}$ and $P$ and $T$ are the pressure and temperature respectively. Since our experiments were carried out in atmospheric pressure, $D \propto$ $T^{3 / 2}$. The second temperature dependent parameter, $c_{s a t}$, may be considered a linear function in the temperature range of this work and may be described as: ${ }^{19,51}$

$c_{s a t}(T)=c_{s a t}\left(T_{a}\right)+\left.\frac{d c_{s a t}}{d T}\right|_{T=T_{a}}\left(T-T_{a}\right)$ 
where $\quad c_{\text {sat }}\left(T_{a}\right)=1.93 \times 10^{-2} \mathrm{~kg} / \mathrm{m}^{3} \quad$ and $\left.\quad \frac{d c_{s a t}}{d T}\right|_{T=T_{a}}=1.11 \times 10^{-3} \mathrm{~kg} /\left(\mathrm{m}^{3} \cdot \mathrm{K}\right)$ at ambient temperature $T_{a}=295 \mathrm{~K}$ and pressure $P_{a}=1 \mathrm{bar}$. Inputting our experimental data for $T$ and $\theta$ in Eq. 2 , 3, 4 we may estimate the evaporation rate of all drops to vary $1.96<-d V / d t<7.55 \mathrm{~nL} / \mathrm{s}$ from low to highest power. Comparison of these theoretical values with the experimental ones presented in Figure 8, varying $3.06<-d V / d t<59.71 \mathrm{~nL} / \mathrm{s}$, shows that the diffusion model clearly under-predicts the evaporation rate and should be revisited for the case of locally heated drops.

(a)

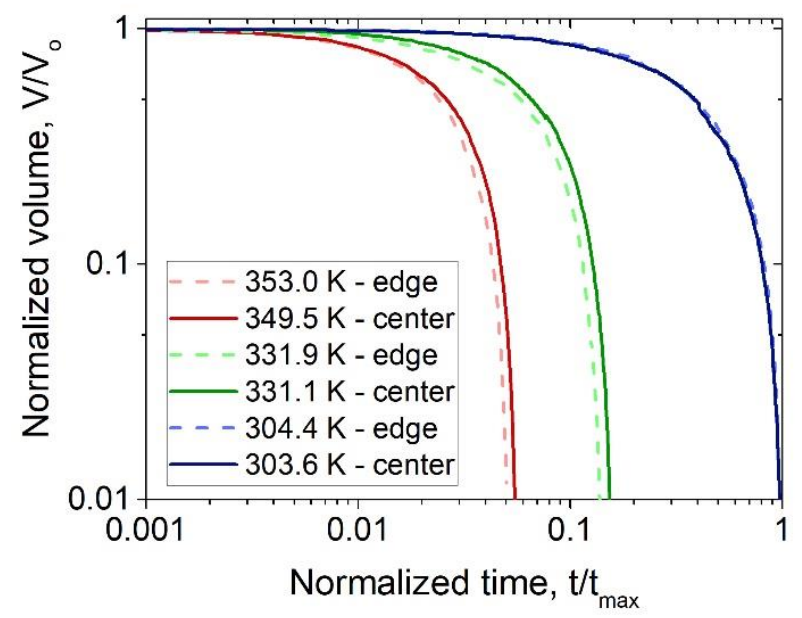

(b)

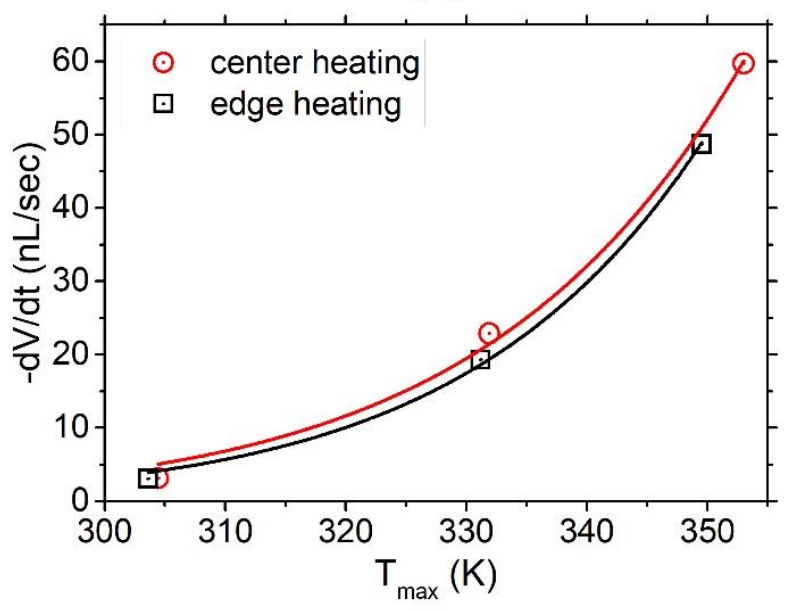

Figure 8: (a) Normalized volume as a function of normalized time for each drop in Figure 5 in log scale.

(b) Effect of heating power and location on the average evaporation rate, $-d V / d t$, of the drops.

\section{CONCLUSIONS}

We examined the effect of local heating on the evaporation of a pure water drop, realized via laser irradiation. Our observations include flow visualization via infrared thermography and optical observation for drop shape changes. We report on the formation of Marangoni flows manifesting as twin vortices with a linear relationship between vortex strength and temperature. The effect of heating location was also examined and found to be paramount to the induction and evolution of the Marangoni flows. Edge 
heating resulted in vortices emerging within the drops at the opposite side of the heating spot and eventually to a periodic increase in the strength of one vortex with a decrease in the other one. On the other hand, center heating led to the formation of twin vortices which tend to travel azimuthally. Eventually, these vortices will either merge and split or shift from one side of the drop to the other in an oscillatory manner, with increasing frequency with heating power. Furthermore, we probed the effect of heating on the evaporation and triple line motion kinetics of a drop. In all cases, local heating was found to promote "stick-slip" evaporation, with the number of depinning events linked with the heating location. In particular, the number of jumps was found to be monotonically decreasing and increasing with power for center and edge heating, respectively, due to a significantly lower surface tension locally in the edge heating case. Additionally, quantification of evaporation kinetics unveiled the significance of heating location on the evaporation process. Overall, these findings show the potential to induce and manipulate Marangoni flows paramount to uniform coating and cooling applications.

\section{ACKNOWLEDGEMENTS}

We are grateful to Mr. Yuji Yasukochi from Spectra Physics Japan for support with experimental setup. This work was financially supported in part by the Core Research for Evolutional Science and Technology project of Japan Science and Technology Agency (JST-CREST).

\section{REFERENCES}

1. de Gans, B.-J.; Schubert, U. S. Inkjet Printing of Well-Defined Polymer Dots and Arrays. Langmuir 2004, 20 (18), 7789-7793.

2. Meyer, S.; Pham, D. V.; Merkulov, S.; Weber, D.; Merkulov, A.; Benson, N.; Schmechel, R. Soluble Metal Oxo Alkoxide Inks with Advanced Rheological Properties for Inkjet-Printed Thin-Film Transistors. ACS Applied Materials \& Interfaces 2017, 9 (3), 2625-2633.

3. Brown, P. O.; Botstein, D. Exploring the new world of the genome with DNA microarrays. Nature Genetics 1999, 21, 33 - 37.

4. $\quad$ Fang, X.; Li, B.; Petersen, E.; Seo, Y.-S.; Samuilov, V. A.; Chen, Y.; Sokolov, J. C.; Shew, C.-Y.; Rafailovich, M. H. Drying of DNA Droplets. Langmuir 2006, 22 (14), 6308-6312. 
5. Askounis, A.; Takata, Y.; Sefiane, K.; Koutsos, V.; Shanahan, M. E. R. "Biodrop" Evaporation and Ring-Stain Deposits: The Significance of DNA Length. Langmuir 2016, 32 (17), 4361-4369.

6. Sefiane, K. On the Formation of Regular Patterns from Drying Droplets and Their Potential Use for Bio-Medical Applications. Journal of Bionic Engineering 2010, 7 (0), S82-S93.

7. Brutin, D.; Sobac, B.; Loquet, B.; Sampol, J. Pattern formation in drying drops of blood. J. Fluid Mech. 2011, 667, 85-95.

8. Bou Zeid, W.; Brutin, D. Influence of relative humidity on spreading, pattern formation and adhesion of a drying drop of whole blood. Colloids and Surfaces A: Physicochemical and Engineering Aspects 2013, 430 (0), 1-7.

9. Askounis, A.; Sefiane, K.; Koutsos, V.; Shanahan, M. E. R. Effect of particle geometry on triple line motion of nano-fluid drops and deposit nano-structuring. Adv. Colloid Interface Sci. 2015, 222, 44-57.

10. Askounis, A.; Sefiane, K.; Koutsos, V.; Shanahan, M. E. R. Structural transitions in a ring stain created at the contact line of evaporating nanosuspension sessile drops. Physical Review E 2013, 87 (1), 012301.

11. Harrington, G. F.; Campbell, J. M.; Christenson, H. K. Crystal Patterns Created by Rupture of a Thin Film. Crystal Growth \& Design 2013, 13 (11), 5062-5067.

12. Hu, H.; Larson, R. G. Analysis of the microfluid flow in an evaporating sessile droplet. Langmuir 2005, 21 (9), 3963-3971.

13. Hu, H.; Larson, R. G. Analysis of the effects of Marangoni stresses on the microflow in an evaporating sessile droplet. Langmuir 2005, 21 (9), 3972-3980.

14. Hu, H.; Larson, R. G. Evaporation of a Sessile Droplet on a Substrate. J. Phys. Chem. B 2002, 106 (6), 1334-1344.

15. Deegan, R. D. Pattern formation in drying drops. Phys. Rev. E 2000, 61 (1), 475-485.

16. Deegan, R. D.; Bakajin, O.; Dupont, T. F.; Huber, G.; Nagel, S. R.; Witten, T. A. Capillary flow as the cause of ring stains from dried liquid drops. Nature 1997, 389 (6653), 827-829.

17. Xu, X.; Ma, L. Analysis of the effects of evaporative cooling on the evaporation of liquid droplets using a combined field approach. Scientific reports 2015, 5.

18. Dash, S.; Garimella, S. V. Droplet evaporation on heated hydrophobic and superhydrophobic surfaces. Physical Review E 2014, 89 (4), 042402.

19. Dunn, G. J.; Wilson, S. K.; Duffy, B. R.; David, S.; Sefiane, K. The strong influence of substrate conductivity on droplet evaporation. J. Fluid Mech. 2009, 623, 329-351.

20. Trouette, B.; Chénier, E.; Doumenc, F.; Delcarte, C.; Guerrier, B. Transient Rayleigh-BénardMarangoni solutal convection. Phys. Fluids 2012, 24 (7), 074108.

21. Dash, S.; Chandramohan, A.; Weibel, J. A.; Garimella, S. V. Buoyancy-induced on-the-spot mixing in droplets evaporating on nonwetting surfaces. Physical Review E 2014, 90 (6), 062407.

22. Pradhan, T. K.; Panigrahi, P. K. Thermocapillary convection inside a stationary sessile water droplet on a horizontal surface with an imposed temperature gradient. Exp. Fluids 2015, 56 (9), 1-11.

23. Hamamoto, Y.; Christy, J. R. E.; Sefiane, K. Order-of-magnitude increase in flow velocity driven by mass conservation during the evaporation of sessile drops. Physical Review E 2011, 83 (5), 051602.

24. Kim, H.; Boulogne, F.; Um, E.; Jacobi, I.; Button, E.; Stone, H. A. Controlled Uniform Coating from the Interplay of Marangoni Flows and Surface-Adsorbed Macromolecules. Phys. Rev. Lett. 2016, 116 (12), 124501.

25. Hegseth, J. J.; Rashidnia, N.; Chai, A. Natural convection in droplet evaporation. Physical Review E 1996, 54 (2), 1640-1644.

26. Sefiane, K.; Moffat, J. R.; Matar, O. K.; Craster, R. V. Self-excited hydrothermal waves in evaporating sessile drops. Appl. Phys. Lett. 2008, 93 (7), 074103.

27. Brutin, D.; Sobac, B.; Rigollet, F.; Le Niliot, C. Infrared visualization of thermal motion inside a sessile drop deposited onto a heated surface. Exp. Therm Fluid Sci. 2011, 35 (3), 521-530. 
28. Chen, P.; Toubal, M.; Carlier, J.; Harmand, S.; Nongaillard, B.; Bigerelle, M. Evaporation of Binary Sessile Drops: Infrared and Acoustic Methods To Track Alcohol Concentration at the Interface and on the Surface. Langmuir 2016, 32 (38), 9836-9845.

29. Girard, F.; Antoni, M.; Faure, S.; Steinchen, A. Evaporation and Marangoni Driven Convection in Small Heated Water Droplets. Langmuir 2006, 22 (26), 11085-11091.

30. MacDonald, B. D.; Ward, C. A. Onset of Marangoni convection for evaporating sessile droplets. J. Colloid Interface Sci. 2012, 383 (1), 198-207.

31. Ristenpart, W. D.; Kim, P. G.; Domingues, C.; Wan, J.; Stone, H. A. Influence of Substrate Conductivity on Circulation Reversal in Evaporating Drops. Phys. Rev. Lett. 2007, 99 (23), 234502.

32. Sáenz, P. J.; Sefiane, K.; Kim, J.; Matar, O. K.; Valluri, P. Evaporation of sessile drops: a threedimensional approach. J. Fluid Mech. 2015, 772, 705-739.

33. Hu, H.; Larson, R. G. Marangoni effect reverses coffee-ring depositions. J. Phys. Chem. B 2006, 110 (14), 7090-7094.

34. Xu, X.; Luo, J.; Guo, D. Criterion for Reversal of Thermal Marangoni Flow in Drying Drops. Langmuir 2010, 26 (3), 1918-1922.

35. Kita, Y.; Askounis, A.; Kohno, M.; Takata, Y.; Kim, J.; Sefiane, K. Induction of Marangoni convection in pure water drops. Appl. Phys. Lett. 2016, 109 (17), 171602.

36. Stauber, J. M.; Wilson, S. K.; Duffy, B. R.; Sefiane, K. Evaporation of Droplets on Strongly Hydrophobic Substrates. Langmuir 2015, 31 (12), 3653-3660.

37. Yang, K.; Hong, F.; Cheng, P. A fully coupled numerical simulation of sessile droplet evaporation using Arbitrary Lagrangian-Eulerian formulation. Int. J. Heat Mass Transfer 2014, 70, 409-420.

38. Bhardwaj, R.; Fang, X.; Somasundaran, P.; Attinger, D. Self-Assembly of Colloidal Particles from Evaporating Droplets: Role of DLVO Interactions and Proposition of a Phase Diagram. Langmuir 2010, 26 (11), 7833-7842.

39. Patil, N. D.; Bange, P. G.; Bhardwaj, R.; Sharma, A. Effects of Substrate Heating and Wettability on Evaporation Dynamics and Deposition Patterns for a Sessile Water Droplet Containing Colloidal Particles. Langmuir 2016, 32 (45), 11958-11972.

40. Colinet, P.; Legros, J. C.; Velarde, M. G. Nonlinear dynamics of surface-tension-driven instabilities; Wiley-VCH Berlin2001; Vol. 527.

41. Garnier, N.; Chiffaudel, A.; Daviaud, F. Hydrothermal Waves in a Disk of Fluid. In Dynamics of Spatio-Temporal Cellular Structures: Henri Bénard Centenary Review, Mutabazi, I.; Wesfreid, J. E.; Guyon, E., Eds.; Springer New York: New York, NY, 2006, pp 147-161.

42. Chandrasekhar, S. Hydrodynamic and Hydromagnetic Stability; Dover Publications1961.

43. Duan, F.; Ward, C. A. Investigation of Local Evaporation Flux and Vapor-Phase Pressure at an Evaporative Droplet Interface. Langmuir 2009, 25 (13), 7424-7431.

44. Shanahan, M. E. R. Simple Theory of "Stick-Slip" Wetting Hysteresis. Langmuir 1995, 11 (3), 1041-1043.

45. Askounis, A.; Sefiane, K.; Koutsos, V.; Shanahan, M. E. R. The effect of evaporation kinetics on nanoparticle structuring within contact line deposits of volatile drops. Colloids and Surfaces $A$ : Physicochemical and Engineering Aspects 2014, 441, 855-866.

46. Bjelobrk, N.; Girard, H.-L.; Bengaluru Subramanyam, S.; Kwon, H.-M.; Quéré, D.; Varanasi, K. K. Thermocapillary motion on lubricant-impregnated surfaces. Physical Review Fluids 2016, 1 (6), 063902.

47. Orejon, D.; Sefiane, K.; Shanahan, M. E. R. Stick-Slip of Evaporating Droplets: Substrate Hydrophobicity and Nanoparticle Concentration. Langmuir 2011, 27 (21), 12834-12843.

48. Picknett, R. G.; Bexon, R. The evaporation of sessile or pendant drops in still air. J. Colloid Interface Sci. 1977, 61 (2), 336-350.

49. Sobac, B.; Brutin, D. Triple-Line Behavior and Wettability Controlled by Nanocoated Substrates: Influence on Sessile Drop Evaporation. Langmuir 2011, 27 (24), 14999-15007. 
50. Poling, B. E.; Prausnitz, J. M.; O'Connell, J. P. The properties of gases and liquids; McGrawHill2001.

51. Sefiane, K.; Wilson, S. K.; David, S.; Dunn, G. J.; Duffy, B. R. On the effect of the atmosphere on the evaporation of sessile droplets of water. Phys. Fluids 2009, 21 (6), 062101-062109.

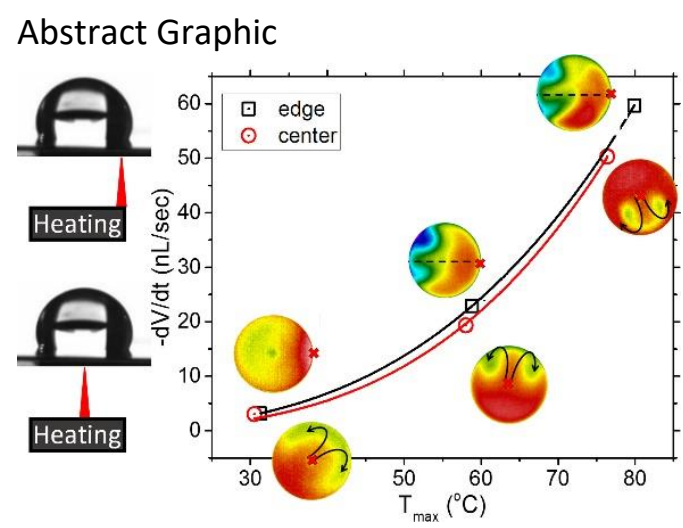

